FORMATION Formation emploi

Revue française de sciences sociales

$110 \mid 2010$

Pêle-mêle

\title{
Les avocats, un marché professionnel déstabilisé
}

Lawyers: a destabilized professional labour market

Rechtsanwälte - ein destabiliserter berufsmarkt

La enseñanza superior en Francia: un espacio segmentado que limita la igualdad de oportunidades

\section{Christian Bessy}

\section{(2) OpenEdition}

Journals

Édition électronique

URL : http://journals.openedition.org/formationemploi/3026

DOI : 10.4000/formationemploi.3026

ISSN : 2107-0946

Éditeur

La Documentation française

Édition imprimée

Date de publication : 15 juin 2010

Pagination : $35-48$

ISSN : 0759-6340

Référence électronique

Christian Bessy, «Les avocats, un marché professionnel déstabilisé », Formation emploi [En ligne],

110 | 2010, mis en ligne le 10 juillet 2012, consulté le 30 octobre 2020. URL : http://

journals.openedition.org/formationemploi/3026 ; DOI : https://doi.org/10.4000/formationemploi.3026

(c) Tous droits réservés 


\title{
Profession
}

\section{Les avocats, un marché professionnel déstabilisé}

\author{
Christian Bessy*
}

\begin{abstract}
De nombreuses incertitudes entourent les carrières des avocats : augmentation des effectifs, plus forte hétérogénéité des conditions d'exercice, concurrence internationale... Elles interrogent l'organisation de leur marché du travail en lien avec la libéralisation du marché des services juridiques.
\end{abstract}

Traditionnellement, dans la profession d'avocat, les jeunes collaborateurs exerçaient sous statut libéral et devaient s'attacher une clientèle pour accéder à une forme d'indépendance, signe d'une insertion professionnelle réussie. C'est l'idée de se faire un nom dans la profession, de se faire connaître, même si certains privilégiés pouvaient bénéficier de la reprise du cabinet de leurs proches. Ce modèle peut conforter des inégalités fortes d'accession à la profession, en l'absence d'un système d'éducation et de formation permettant à tous d'acquérir au moins des compétences juridiques.

La démocratisation de l'enseignement supérieur en général, et des facultés de droit en particulier, a permis, de ce point de vue, une ouverture de la profession à des classes moins fortunées dans une période de développement des activités juridiques. Depuis les années 70,

\footnotetext{
${ }^{1}$ Ce texte reprend le chapitre d'un rapport commandité par le Conseil national des Barreaux (CNB par la suite) sur l'évolution de la réglementation de la profession des avocats (Favereau et alii, 2008). Il a également bénéficié du support du programme «Les appuis sociaux de l'entrepreneuriat», financé par l'ANR. L'auteur remercie Fabienne Berton, Pierre-Paul Zalio, et les referees anonymes de la revue pour leurs critiques stimulantes d'une première version de ce texte.
}

on assiste à un accroissement des activités judiciaires et juridiques et du nombre d'avocats, ainsi qu'à la diversification de leur recrutement (Vauchez et Willemez, 2002). Cet accroissement bute aujourd'hui sur certaines limites en raison des difficultés durables d'intégration professionnelle des « nouveaux arrivants », de

* Christian Bessy est économiste du travail et chargé de recherche CNRS à l'IDHE-ENS (Institutions et Dynamiques Historiques de l'Économie) - École normale supérieure de Cachan. Habilité à diriger des recherches, il enseigne actuellement au CNAM /Conservatoire national des arts et métiers) et à l'ENS de Cachan. II est spécialiste de l'analyse économique des institutions et du marché du travail. II a débuté ses recherches au Centre d'études de l'emploi en consacrant une thèse à la réglementation du licenciement économique. II a poursuivi sur l'analyse des pratiques de recrutement et s'est intéressé en parallèle au droit de la propriété intellectuelle. Ses recherches actuelles portent sur le rôle du droit dans la régulation des activités économiques et sur les dynamiques d'innovation. II a publié récemment "La contractualisation de la relation de travail » (2007) aux éditions LGDJ. 
nombreux collaborateurs quittant la profession au bout d'une dizaine d'années, notamment les femmes.

Le courant institutionnaliste en économie a depuis longtemps analysé ce problème de la promotion régulière de collaborateurs à un statut de propriétaires de cabinets (seuls ou associés), ou encore de salariés ayant une certaine sécurité d'emploi, en référence au fonctionnement d'un marché du travail basé sur la mobilité de professionnels dotés de qualifications transférables assujetties à des normes de qualité et qui présupposent une certaine uniformité des postes de travail d'une entreprise à une autre (Kerr, 1954). Plus récemment, un auteur comme D. Marsden (1999) a montré qu'un « marché professionnel du travail », qui représente un mode d'organisation du marché du travail alternatif à un « marché interne d'entreprise », est une construction fragile faite à la fois de relations de coopération et de concurrence entre employeurs. Qui va payer pour la formation des jeunes professionnels ? Une part de la compétence peut être fournie par l'État (formation initiale), mais une part essentielle de la compétence ne peut s'acquérir qu'au sein et au contact des entreprises employeuses. Une entreprise peut être tentée de ne pas contribuer à ces dépenses et de débaucher les travailleurs formés par les concurrents. La difficulté est alors la même que celle qui pénalise la fourniture de « biens publics ». Ou encore, et c'est la solution concurrentielle de la théorie du capital humain de G. Becker (1964), c'est au salarié qu'il revient de financer la formation du capital humain général (au

\section{Encadré 1 \\ La profession d'avocat}

En janvier 2007, on comptait 47665 avocats, selon les chiffres du ministère de la Justice, avec pratiquement autant d'hommes que de femmes. À titre de comparaison, ils étaient 34078 avocats en 1998, soit une croissance de $40 \%$ en dix ans. Pratiquement un avocat sur deux exerce en lle-de-France. La répartition des avocats selon le mode d'exercice, en 2007, s'effectue presque de façon équilibrée entre la collaboration, l'exercice individuel et l'association, structure qui associe exclusivement des avocats (on parle alors d' "associés") et qui peut relever de différents statuts juridiques.

Le " collaborateur » est le plus souvent un jeune avocat qui travaille de façon autonome sur les dossiers qui lui sont confiés. Sa rémunération s'effectue sous forme de rétrocession d'honoraires, au sens où l'avocat associé rétrocède une partie de ses honoraires en contrepartie du travail de son collaborateur. Au moment de notre enquête auprès d'une trentaine d'avocats, le montant de cette rétrocession pouvait aller de 3000 euros brut par mois à plus de 6000 euros lors de leur première embauche. Notons aussi que le statut de collaborateur peut s'exercer sous un contrat de travail moyennant le versement régulier d'un salaire. Les avocats salariés (représentant 6,8 \% des effectifs d'avocats en 2007), ne peuvent pas constituer de clientèle personnelle, à la différence des collaborateurs dits " libéraux ».

Le décret du 21 décembre 2004 a modifié les règles de la formation initiale des avocats. Une fois entré au Centre régional de formation à la profession d'avocat, le futur avocat bénéficie d'une formation qui se déroule désormais sur 18 mois, incluant un stage, formation à l'issue de laquelle est présenté le Certificat d'aptitude à la profession d'avocat (CAPA). Reçu au CAPA, I'avocat prête serment et s'inscrit à I'ordre de son choix. Ces nouvelles règles modifient à la baisse la durée des études supérieures ainsi que celle du stage nécessaire à l'entrée dans la profession. En 2008, 3056 élèves avocats fréquentaient les centres de formation, contre 2547 en 2000 . Notons qu'il existe d'autres voies d'accès à la profession d'avocat mais qui restent très marginales, comme la possibilité pour les juristes d'entreprise de s'inscrire au Barreau au bout de huit années d'activité. II faut compter aussi sur l'inscription des avocats étrangers, dont le nombre ne cesse d'augmenter, pour atteindre 1427 avocats en 2007, soit $3 \%$ de l'effectif total.

NB: Les chiffres donnés ici proviennent de l'Observatoire du Conseil national des Barreaux (CNB, 2008). 
sens de transférable dans une autre entreprise). Cette solution se traduit par une rémunération inférieure à la « productivité » du travailleur. Ce dernier peut alors être découragé d'entrer dans la profession s'il n'obtient pas de fortes garanties sur ses chances de promotion ultérieure. Dans les deux cas, la concurrence entre cabinets peut alors déboucher sur une configuration peu favorable à tous. Il faut alors inventer collectivement une réglementation, en fixant un certain niveau de rémunération pour les jeunes collaborateurs et en sanctionnant les pratiques de débauchage opportunistes, ce qui constitue pour Marsden (op. cité) les deux règles fondamentales de la stabilisation d'un marché professionnel.

L'objectif de cet article est de montrer que la profession des avocats ne parvient plus véritablement à gérer ce problème inter-temporel lié à la formation et à la carrière des jeunes avocats. Pour cela, nous allons analyser certains facteurs à l'origine de cette déstabilisation du marché professionnel, et en particulier la plus grande variété des cabinets. Cette dernière résulte du développement des cabinets d'affaires, plus orientés vers le conseil juridique que vers le contentieux, et de l'internationalisation de leurs activités.

Pour bien comprendre quels sont les enjeux autour de la formation des collaborateurs, nous examinerons le développement du statut de l'avocat salarié (première partie). Cette analyse va permettre d'éclairer les spécificités françaises en la matière et de bien appréhender une contrainte forte de l'installation des collaborateurs, à savoir la possibilité qui leur est accordée de développer leur propre clientèle. En croisant cette caractéristique avec la politique des cabinets concernant la rémunération de leurs collaborateurs, nous en tirons analytiquement quatre modèles de collaboration. Dans une deuxième partie, nous abordons l'émergence de la figure du « collaborateur entrepreneur », en lien avec le développement des grands cabinets d'affaires qui impulsent un nouveau modèle rendant plus incertaines les carrières des collaborateurs. La troisième partie est alors consacrée à la limitation de leurs perspectives de carrière ; elle étudie leurs difficultés d'accès à l'indépendance économique et les freins à leur mobilité.

D'un point de vue empirique, nous prenons appui sur les entretiens semi-directifs menés en 2007-2008 auprès d'une trentaine d'avocats appartenant à des cabinets différents en termes de structures d'exer- cice (taille, Paris/province...) et de domaines du droit (droit des affaires, droit social et droit de la famille). Les entretiens auprès d'avocats travaillant dans des cabinets d'affaires sont ici particulièrement mobilisés ; en effet, ces cabinets ont impulsé un nouveau modèle de collaboration qui remet en cause le modèle traditionnel de l'apprentissage.

\section{DES PROBLÈMES DE COORDINATION LIÉS AU FINANCEMENT DE LA FORMATION}

Pour étudier ces problèmes de coordination, nous allons partir du choix, pour un cabinet, entre un contrat de collaboration salarié et un contrat non-salarié. Au début des années 2000, l'étude de Vauchez et Willemez (2002) avait souligné la proportion relativement importante des avocats salariés $(7,5 \%$ en 2000$)$. À l'encontre des prévisions faites par ces sociologues, le nombre d'avocats salariés est aujourd'hui en légère diminution. Avant d'analyser ce retournement, dans la deuxième partie, revenons d'abord à la phase de développement du statut de l'avocat salarié.

\section{Le droit social incite au salariat}

Initialement, le statut d'avocat salarié naît de la fusion, en 1991, des avocats et des conseils juridiques, ces derniers ayant largement recours au salariat. Un deuxième élément d'extension de ce statut tient à une évolution de la jurisprudence poussant à la requalification en contrat de travail de contrats de collaboration libérale ; cette évolution sera contrariée ou au contraire consolidée suivant la succession des textes législatifs, liée à l'alternance politique ${ }^{2}$.

Le recours au salariat s'est particulièrement développé dans les cabinets spécialisés en droit social. La protection du collaborateur est ainsi recherchée par l'adoption d'un statut de salarié. Ce statut est défini par le droit du travail et la convention collective

\footnotetext{
2 Ainsi, la loi Madelin de 1994 a introduit la présomption de nonsalariat, rendant ainsi plus difficiles les requalifications en contrat de travail. Cette orientation a été remise en cause par le gouvernement socialiste puis remise au goût du jour par la loi Dutreil de 2005.
} 
signée le 17 février 1995, pour toutes les dispositions autres que celles instaurées par la loi du 30 décembre 1990 et le décret du 27 novembre 1991 (relatif à la fusion). Mais ce statut peut aussi s'expliquer par la perte d'une certaine indépendance du fait d'une intégration poussée de l'avocat dans la division du travail des organisations qui se développent et se spécialisent dans différents domaines. Cette division du travail peut conduire l'avocat à ne plus être maître de l'argumentation qu'il développe et des conseils qu'il donne, ou encore de ne plus être déchargé d'une mission qu'il estime contraire à ses valeurs.

C'est justement cette intégration de collaborateurs libéraux plus poussée à l'organisation, leur empêchant de constituer une clientèle, qui a été à l'origine de nombreux contentieux qui ont débouché sur des requalifications en contrat de travail. E2 (avocate en droit social qui a exercé de grandes responsabilités professionnelles) retrace l'origine de ces contentieux en repartant de la distinction entre les deux statuts de la collaboration :

"Le grand principe est que, compte tenu de la nature de la profession réglementée que nous exerçons, il ne peut pas y avoir de lien de subordination dans l'exercice de son métier, mais uniquement dans les conditions de travail. Ça, c'est la première des distinctions. En effet, dans le contrat de travail des avocats salariés, l'employeur, donc le patron, peut exercer le lien de subordination sur les conditions de son travail. Par ailleurs, deuxième critère, l'employeur, le " patron" pour une collaboration libérale, doit donner les moyens au collaborateur libéral de développer sa clientèle. Et autour de ça s'est articulé, à mon avis, un double mouvement : un mouvement d'explosion du droit, donc de la profession, avec des disparités de plus en plus grandes entre les avocats, à savoir toute une réflexion sur le point de savoir s'il fallait ou non que le jeune paye sa formation - ça c'est une idée personnelle parce que disons que, traditionnellement, les avocats de souche étaient très attachés à la notion de stage, de maître stagiaire, et le défraiement était plus le principe qu'une rémunération. Puis, compte tenu de l'explosion démographique, les jeunes avocats, dès lors qu'ils ne pouvaient pas développer une clientèle personnelle, ont été amenés à demander des fixations de rétrocession minimale, d'où un travail sur la question de savoir si le collaborateur libéral avait réellement les moyens de développer sa clientèle. "

Nous ne reviendrions pas ici sur la distinction entre la « subordination dans l'exercice de son métier » et la « subordination dans les conditions de travail », pour nous concentrer sur l'explication des contentieux, donnant lieu à requalification, avancée par E2 ${ }^{3}$. En fait, avec l'explosion démographique des avocats et la diversification de leurs conditions d'exercice, c'est le modèle même de leur formation par un long apprentissage chez un « maître » qui est remis en cause, ainsi que leur rémunération qui était relativement faible et qui servait avant tout à couvrir les frais professionnels des jeunes collaborateurs. C'est dans ce sens que l'avocate parle de « défraiement ».

\section{La double composante} des compétences des jeunes avocats

On touche ici au problème central de la relation de travail, à savoir le financement de la formation professionnelle des jeunes, problème qui se complexifie dans le cas des professions où la réussite de la carrière passe par la mise à son compte ou l'association. Dans la mesure où les clauses de non-concurrence sont interdites, le jeune avocat peut quitter la structure qui l'a hébergé pour s'installer, avec sa part de clientèle, dans une autre structure. Dès lors, la constitution de cette clientèle va être une source d'antagonismes entre les intérêts en présence.

Ce que nous dit l'avocate dans l'extrait précédent, c'est que les jeunes avocats, ne pouvant pas développer une clientèle personnelle, ont demandé une compensation sous forme d'une rétrocession minimale d'honoraires. Signalons que, dès la "fusion », le décret de 1991 prévoyait que le règlement intérieur de chaque barreau pourrait fixer un barème de rétrocession minimale d'honoraires. Dans les faits, beaucoup de barreaux provinciaux (versus l'ordre parisien), n'ont pas adopté de tels minima et, lorsque ces derniers existent, ils sont généralement faibles (CNB, 2005). Cela a été à la source de litiges témoi-

${ }^{3}$ Pour de plus amples développements, voir Barthélémy et alii (2008). 
gnant de l'éloignement du modèle traditionnel de l'apprentissage des jeunes avocats dans lequel, comme nous l'a rappelé notre interlocutrice, leur rémunération était plus considérée comme un "défraiement» que comme une véritable rémunération. Ce modèle aussi leur permettait progressivement de s'attacher une clientèle et donc d'exercer ensuite, s'ils le voulaient, de façon indépendante. Les comportements trop opportunistes des jeunes avocats (démission une fois formé et mobilisation ensuite d'une partie de la clientèle $\mathrm{du}$ cabinet formateur qui ne récupère donc pas son « investissement »), ou de ceux qui les débauchent prématurément doivent être sanctionnés par la profession au nom d'une concurrence déloyale.

Or, nombre de nos interlocuteurs dénoncent les pratiques intempestives de débauchage de certains cabinets d'affaires anglo-saxons qui, du fait de leur taux de rentabilité élevé, ont accordé des rétrocessions importantes pour attirer les meilleurs éléments sans avoir à supporter les coûts de formation professionnelle. Cela s'est propagé dans d'autres domaines du droit, participant de la sorte à un effet de chaises musicales au sein des cabinets français.

\section{Quatre modèles de collaboration}

La formation en compétences transférables comprend donc une double composante : compétences techniques, attachement d'une clientèle. Et c'est l'absence de cette seconde composante qui peut servir d'argument au collaborateur libéral pour justifier une requalification de son contrat.

Pour revenir à la prégnance du statut d'avocat salarié, on peut alors l'expliquer comme un choix de gestion des cabinets permettant à la fois d'éviter les contentieux liés à la « requalification » des contrats et de fidéliser un personnel formé à leurs frais. Cette solution a également pour avantage de rationaliser l'organisation du travail dans des structures qui s'étoffent et qui diversifient leurs offres de services, tout en restant spécialisées dans un domaine du droit qui évolue en permanence (droit social, ou encore NTIC - nouvelles technologies de l'information et de la communication - et propriété intellectuelle). Cette stratégie de niche, offrant des services juridiques pointus à une clientèle qui tourne, permet de se protéger de la concurrence des autres cabinets. En effet, les collabo- rateurs spécialisés dans un domaine et investis dans des dispositifs organisationnels spécifiques ont plus de mal à redéployer leurs compétences dans d'autres domaines du droit ou d'autres organisations.

Notons que les travaux de E. Goreman (1999) montrent que les law firms ${ }^{4}$ américaines organisées par départements spécialisés, qui tentent de s'éloigner du modèle « up or out $\|^{5}$, cherchent aussi à stabiliser une partie de leur personnel en l'employant sous un contrat de travail. Ce personnel salarié regroupe des avocats confirmés et des collaborateurs qui ont dépassé la période probatoire pour devenir associés, période qui dure en moyenne une dizaine d'années après l'entrée dans le cabinet.

Pour récapituler nos arguments analytiques, on peut distinguer quatre modèles de collaboration (collaborateur innovant, traditionnel, entrepreneur, exploité) en croisant deux variables de gestion des collaborateurs : le montant de la rémunération (ou rétrocession d'honoraire), en distinguant schématiquement deux modalités (élevée : + de 5000 euros brut, et faible : entre 3000 et 4000 euros brut), la possibilité ou non de développer des compétences transférables. Ces quatre modèles, synthétisés dans le tableau $\mathbf{1}$, renvoient à notre travail antérieur de typologie des cabinets dans lequel nous avons présenté une caractérisation de leurs activités suivant deux critères : degré de couplage conseil/contentieux et degré de co-production du service juridique entre l'avocat et son client (Favereau et alii, 2008). Dans cette recherche, ces modèles d'activité sont associés à différentes « conventions de qualité » (Eymard-Duvernay et alii, 2006) pour mettre l'accent sur leur dimension normative mêlant à la fois efficacité et équité, et formes d'évaluation (des produits et des personnes) qui soutiennent ces modèles, et donc sur la stabilisation des attentes des uns vis-à-vis des autres, en particulier des attentes de carrière des collaborateurs.

\footnotetext{
${ }^{4}$ Expression consacrée dans le domaine pour désigner les grands cabinets américains qui offrent des prestations juridiques. C'est difficilement traduisible en français car jusqu'à une période récente les cabinets d'avocats n'étaient pas considérés comme des entreprises ayant une « activité économique».

${ }^{5} \mathrm{Ce}$ modèle renvoie à l'idée qu'un collaborateur qui n'accède pas à l'association dans son cabinet (up) se voit obligé de présenter sa démission (out). Cette norme de mobilité a fait l'objet de nombreuses analyses, à partir des années 90, aux États-Unis, dans les entreprises offrant des services professionnels (Malos et Campion, 1995).
} 
Tableau 1

Quatre modèles de collaboration

\begin{tabular}{|c|l|l|}
\hline $\begin{array}{c}\text { Développer des Rémunération }\left(^{*}\right) \\
\text { compétences transférables }\end{array}$ & \multicolumn{1}{|c|}{ Élevée } \\
\hline Possible & $\begin{array}{l}\text { Collaborateur innovant } \\
\text { Cabinets innovants et qui forment } \\
\text { leurs collaborateurs } \\
\text { Formation spécifique et statut de salarié }\end{array}$ & $\begin{array}{l}\text { Collaborateur traditionnel } \\
\text { Cabinets traditionnels basés } \\
\text { sur l'apprentissage sur le tas } \\
\text { et le "défraiement » }\left(^{* *}\right)\end{array}$ \\
\hline Pas possible & $\begin{array}{l}\text { Collaborateur entrepreneur } \\
\text { Grands cabinets d'affaires diversifiés } \\
\text { qui ont tendance à débaucher } \\
\text { plutôt que de former } \\
\text { Formation spécifique et statut de salarié }\end{array}$ & $\begin{array}{l}\text { Collaborateur « exploité » } \\
\text { Cabinets qui instaurent une relation } \\
\text { de travail peu coopérative } \\
\text { Risque de requalification du contrat }\end{array}$ \\
\hline
\end{tabular}

Source : enquête des auteurs.

(*) Élevée : plus de 5000 euros brut. Faible : entre 3000 et 4000 euros brut.

$(* *)$ : Faible rémunération qui servait avant tout à couvrir les frais professionnels des jeunes collaborateurs.

Un premier modèle, dit du « collaborateur innovant», renvoie à des cabinets dans lesquels les collaborateurs sont bien formés et peuvent s'attacher une clientèle, tout en percevant des rémunérations élevées. Celles-ci permettent d'attirer et de récompenser des talents. Cela suppose que les cabinets soient suffisamment innovateurs pour opérer auprès d'une clientèle prête à verser des honoraires conséquents. Ce sont des cabinets dits de « haute couture », ou encore de « niche», articulant fortement les activités de consultation juridique (par la suite « conseil ») et celles inhérentes au traitement des affaires impliquant un contentieux (par la suite « contentieux »). La complexité de la prestation juridique à destination d'une clientèle qui tourne peut conduire les plus grands cabinets de niche à opter pour une collaboration salariée.

Le second modèle, du « collaborateur traditionnel », correspond à l'apprentissage traditionnel du métier d'avocat au cours duquel le cabinet s'investit dans la formation générale du collaborateur mais en proposant des rétrocessions d'honoraires plus faibles (il s'agit plutôt de défraiement). Cette rémunération relativement faible peut également s'inscrire dans une forme de désintéressement liée à la défense de causes politiques.

Les deux autres formes de collaboration sont plus directement liées aux pressions du marché et concernent des cabinets qui capitalisent la clientèle.
Les grands cabinets d'affaires diversifiés et spécialisés par département misent sur un « collaborateur entrepreneur », qui doit démarcher les clients. Ces cabinets peuvent débaucher de jeunes talents déjà formés en leur accordant des rétrocessions élevées. Comme pour les plus grands cabinets de niche, l'investissement dans des formations plus spécifiques propres à un domaine du droit peut conduire à l'adoption du statut de collaborateur salarié.

La dernière configuration, celle du collaborateur « exploité », ne constitue pas véritablement un « modèle de collaboration » car elle n'est pas propice à la coopération. Il s'agit de petites structures subissant fortement la concurrence des autres types de cabinet. Elles sont donc peu à même de fournir des compétences transférables aux collaborateurs, en particulier leur permettre de développer une clientèle, et de bien les rémunérer. «L'exploitation » du collaborateur peut être à l'origine de litiges ou d'un turnover élevé.

Ces quatre modèles de collaboration vont nous servir de grille d'analyse pour aborder l'évolution et la déstabilisation du marché professionnel traditionnel. À ce stade de notre développement, le contraste entre ces quatre modèles fournit un aperçu des tensions à gérer au sein de la profession et des problèmes de coordination en matière de formation auxquels elle est actuellement confrontée. 


\section{LE RETOUR AU STATUT DE COLLABORATEUR LIBÉRAL}

Malgré les tensions au sein de la profession, suscitées par les tarifs de rétrocession minimale, le cas des avocats collaborateurs libéraux a été cité en exemple pour les professions libérales, lors de la préparation de la « loi Dutreil» de 2005 sur la modernisation de l'entreprise. Cette loi a défini de façon précise le contrat de collaboration libérale non salariée afin de créer des emplois supplémentaires, au sein des professions ne connaissant pas ce type de contrat, et de permettre l'intégration de jeunes professionnels libéraux au sein de structures dans lesquelles ces derniers auraient été formés. C'est aussi évidemment pour permettre au collaborateur de pouvoir un jour s'installer. Le CNB (Conseil national des barreaux) a ainsi modifié l'article 14 du Règlement intérieur national sur le statut de la collaboration libérale, afin de concrétiser les dispositions de la loi Dutreil de 2005 (article 18). Ces modifications ont notamment porté sur l'obligation, pour le cabinet, de mettre des moyens à la disposition du collaborateur pour développer sa clientèle personnelle, sans contrepartie financière, et de faciliter ses activités en matière d'aide juridictionnelle, commission d'office, garde à vue et consultations gratuites ${ }^{6}$. Ces activités sont peu rémunératrices pour l'avocat mais permettent de contribuer à l'accès de tous à la justice.

Nous allons justement montrer que ce modèle d'intégration de jeunes professionnels libéraux a perdu de ses vertus en raison des difficultés de carrière rencontrées par certains d'entre eux. Ces difficultés d'ailleurs émergent parallèlement au recul du statut de collaborateur salarié. En effet, depuis cinq ans, on observe une baisse de la proportion d'avocats salariés. Elle passe de $8,6 \%$ en 2002 à $6,8 \%$ en 2007 , au sein

\footnotetext{
${ }^{6}$ L'aide juridictionnelle a pour objectif de satisfaire les demandes adressées par des personnes physiques, dont les ressources sont insuffisantes, portant sur l'application de leur droit, de faciliter la connaissance par toute personne de ses droits, d'améliorer l'indemnisation des avocats prêtant leur concours aux justiciables (réforme de 1992). Elle a été complétée, dès 1993, par l'aide à l'intervention de l'avocat au cours de la garde à vue. Contrairement à l'aide juridictionnelle où le client peut choisir son avocat, la commission d'office est la procédure par laquelle le Bâtonnier (qui préside l'ordre professionnel) désigne un avocat lorsqu'un particulier doit comparaître devant une juridiction pénale et qu'il ne connaît pas d'avocat.
}

d'une évolution d'ensemble qui voit toujours croître le nombre d'avocats : de 37708 au $1^{\text {er janvier } 2002 \text { à }}$ 46636 en 2007 ( $4 \%$ par an). Cette baisse relative de la proportion d'avocats salariés est liée à différents facteurs.

Il ne faut sans doute pas sous-estimer la loi Dutreil qui, comme nous venons de le rappeler, a voulu favoriser le développement de la collaboration libérale. Condition nécessaire, mais sans doute pas suffisante. On peut faire référence à un changement plus idéologique ou plus lié aux modes de développement des activités des cabinets d'affaires, à savoir l'émergence $\mathrm{du}$ « collaborateur entrepreneur ». Cette nouvelle figure de la collaboration s'inscrit dans la diffusion croissante des normes managériales, en œuvre dans les grandes entreprises, au sein des cabinets d'affaires. Cette diffusion est liée à une conception du service juridique qui correspond à une forme de co-production entre l'avocat et son client, remettant en cause l'indépendance professionnelle du premier (Karpik, 2007). Elle se développe en parallèle avec la demande croissante de services juridiques individualisés à l'entreprise, comme la multiplication des opérations de fusions et acquisitions d'entreprises. Ce changement du rôle professionnel des avocats, qui va au-delà des seuls avocats d'affaires, se retrouve dans divers pays caractérisés par des traditions juridiques différentes, comme le montre Lazega (2001) pour les « law firms » américaines.

\section{Changement idéologique : le collaborateur entrepreneur}

Pour E3 (associée en droit social dans un cabinet d'affaires anglo-saxon), les avocats salariés n'ont été qu'une scorie des anciens cabinets de conseil juridique, qui tend aujourd'hui à disparaître, car, pour elle, un avocat c'est avant tout un « indépendant ». Par ailleurs, elle souligne le fait que les collaborateurs, dans son cabinet, surtout en droit social, ont toutes les possibilités de développer leur clientèle personnelle, la structure leur en offrant les moyens :

\section{"Et donc, il y a un défraiement si le collabora- teur mobilise les ressources?"}

«Non, c'est tout à fait dans la mentalité de la maison et ça fait partie du rôle du jeune aussi 
parce que on veut des gens qui sont des entrepreneurs. L'avocat, ce n'est pas juste quelqu'un qui traite les dossiers. Il faut aussi savoir satisfaire ses clients, garder un relationnel, aller chercher des dossiers. Si vous dites aux jeunes qu'ils n'ont pas le droit de le faire parce que vous voulez qu'ils ne travaillent que pour vous, comment vous voulez qu'ils développent ça. »

"Alors justement, parce qu'on nous a dit avant - bon ils ont le droit de le faire, ça on le sait, mais que, en pratique, par rapport à ce débat "libéral ou salarié ", ils ont du mal à le faire, ils ne peuvent pas le faire. Alors apparemment chez vous c'est le contraire..."

« Je peux vous assurer que... On a fait des groupes de discussion avec les collaborateurs tous niveaux d'ancienneté et pratique confondus, vous êtes étonnés de voir comment pour certains c'est fun et ils ont envie de le faire parce que ça arrondit les fins de mois, et il y en a d'autres qui disent "Ah non, moi ça ne m'intéresse pas. Je suis très bien payé. Je n'ai pas envie de faire ça en plus" "».

" Et au niveau global, par rapport à cette idée que ce serait quand même difficile. Alors est-ce que c'est une exception de votre cabinet ?"

"Tout dépend de ce que l'on veut! Je ne sais pas. Moi j'ai été 10 ans chez Symphonie et c'est ce qu'on appelle la "clientèle perso" ...»

"Chez Symphonie, on pouvait la développer aussi?"

"Symphonie est un cabinet d'affaires et j'ai eu beaucoup de clients persos. Certains sont devenus des clients cabinet le jour où je suis devenue associée. F. c'est un cabinet d'affaires et je peux vous assurer que dans le groupe du droit du travail ils ont tous des prud'hommes à titre perso, des transactions qu'ils négocient, des dossiers qui... ça c'est, vraiment, c'est l'avocat qui a envie de le faire. Alors il y en a qui n'ont pas envie! Il $y$ en a qui trouvent que les journées sont assez longues, ils ne vont pas se rajouter du boulot, moi je respecte ».

Pour les collaborateurs libéraux, la possibilité de développer une clientèle personnelle n'est pas simple- ment une obligation à la charge du cabinet, c'est devenu aussi une obligation pour le collaborateur qui doit faire preuve d'un esprit d'entrepreneur. Cela ne semble pas propre à ce cabinet, car cette obligation se retrouve dans d'autres structures identiques. Bien que ce genre d'exercice à titre personnel soit d'une certaine façon contrôlé au sein des cabinets afin qu'il ne se réalise pas au détriment de leurs propres affaires, il faut sans doute retenir cette rhétorique du petit entrepreneur qui développerait une compétence pour prospecter et s'attacher une clientèle pour son compte et celui du cabinet.

Dans d'autres grands cabinets d'affaires caractérisés par une organisation très intégrée, il n'est pas question pour le collaborateur de développer sa « clientèle perso ». Il doit en permanence prospecter la clientèle et participer à l'accroissement de la part de marché chez les clients qui, par ailleurs, ne veulent pas être tributaires d'un seul cabinet d'avocats. Dans cette configuration, le collaborateur n'a aucun droit sur la clientèle ou d'autre actifs immatériels qu'il contribue à développer, ni même une quelconque garantie sur la possibilité de devenir associé. L'accès à l'association est de plus en plus long et incertain. Pour le collaborateur, l'alternative est de devenir salarié du cabinet, à condition que son expertise dans un domaine soit reconnue, ou, alors, chercher à être associé dans un autre cabinet. Ainsi, dans un même cabinet, des formes d'intégration de certains collaborateurs peuvent côtoyer une rotation importante de l'autre partie du personnel.

En résumé, pour le « collaborateur entrepreneur », la seule assurance fixée à l'embauche, c'est la rétrocession, dont le montant varie suivant son pouvoir de négociation sur le «marché du travail ». On peut faire l'hypothèse que c'est l'institution de cette norme, de ce nouveau modèle de collaboration et de fonctionnement du marché du travail associé qui est en jeu depuis le début des années 2000 et qui a conduit les cabinets à se détourner du statut de collaborateur salarié. Ce statut apparaît de plus en plus comme une exception en œuvre dans les grandes structures d'exercice développant des spécialisations pointues.

Dans certains cabinets, le passage au statut de collaborateur libéral s'est réalisé de façon abrupte, comme dans ce petit cabinet d'affaires parisien qui comptait beaucoup de collaborateurs salariés : 
«Il y a cinq ans, ils ont négocié pour passer les collaborateurs en libéral. Ils ont cherché à écrémer la structure en allant jusqu'à licencier et passer des transactions, car le statut de libéral est moins contraignant. »

Notre interlocuteur, jeune collaborateur en droit social, explique ce brusque changement de cap par le fait que, pour un cabinet, cette solution est plus souple en termes de gestion - le cabinet peut rompre la relation quand il veut - et moins coûteux, car les charges sociales sont directement supportées par le collaborateur. Cette recherche de flexibilité peut être reliée à la diversification croissante des cabinets qui multiplient leur intervention dans les différents domaines du droit. Lorsqu'un domaine d'activité est en chute, le cabinet peut alors plus facilement se séparer des collaborateurs que dans le cadre d'une procédure de licenciement. Cette facilité de rupture du contrat propre au statut libéral limite également les coûts de gestion du personnel par rapport au statut salarié.

Du côté du collaborateur, le statut de libéral exerce un effet d'entraînement, le valorise, par rapport à celui de la dépendance salariale, mais ce statut ne répondant plus aux attentes des collaborateurs, il est remis en cause.

\section{Un statut qui ne répond plus aux attentes des collaborateurs}

Contrairement à ce qu'avançait E3 précédemment, il semble que nombre de collaborateurs n'ont pas les moyens de développer leur propre clientèle, en particulier dans les cabinets d'affaires, comme nous l'ont déclaré les quatre collaborateurs que nous avons rencontrés (de 2 à 6 ans d'ancienneté dans des cabinets d'affaires de taille moyenne). Ils remettent en cause le statut de collaborateur libéral qui n'assure plus aujourd'hui la transition vers l'association ou l'indépendance. Par ailleurs, ils dénoncent la dépendance totale du collaborateur à son associé. Cette dépendance est rapportée à l'organisation même des cabinets d'affaires qui prend plus appui sur des équipes constituées par chaque associé, ayant sa propre clientèle, que sur une organisation bien structurée en référence à des départements spécialisés. Dans cette configuration peu intégrée, la clientèle appartient à l'associé et non au cabinet, à la différence de tous les grands cabinets dont la réputation s'appuie sur leur image de marque et dont la clientèle est « socialisée ».

Les collaborateurs interrogés dénoncent aussi la dégradation des conditions d'exercice de leur métier? Ils se sentent sous pression et dévalorisés par des associés qui sont " caractériels et qui ne pensent qu'à gagner de l'argent $»$ en traitant des affaires très lucratives de fusion-acquisition d'entreprises. Cette démesure entre les rétrocessions des collaborateurs et les montants des heures facturées par le cabinet accroît leur sentiment d'injustice.

Cette rémunération peut être considérée comme insuffisante du fait d'un travail effectué plus intense que d'habitude. L'injustice se situe alors dans le manque de reconnaissance des efforts consentis par les collaborateurs, pouvant les amener à "passer des nuits et des week-end sur leurs dossiers ». Ceux-ci dénoncent alors le manque d'esprit d'équipe et de reconnaissance du travail de chacun, qui peut conduire à la démission des collaborateurs. Cette rémunération peut être aussi considérée comme satisfaisante et même substantielle, dès lors que le travail supplémentaire est payé. Mais cette prestation bien rémunérée s'opère au détriment du sens donné au travail et de conditions de travail difficiles (horaires sans fin et travail le week-end) qui poussent les collaborateurs à changer de métier bien qu'ils soient bien rémunérés, comme cette collaboratrice qui en est à sa deuxième expérience dans un cabinet d'affaires :

"Je n'y passe pas toutes mes nuits et week-end. Il ne faut pas exagérer. Mais je fais cela pendant 5 ou 6 ans et puis après je passe à autre chose. C'est vrai, je suis bien payée. Je gagne 5200 euros brut auxquels il faut enlever près de la moitié pour avoir le net... Je n'ai pas d'intéressement en fonction des heures travaillées. C'est le contrat standard de collaboration... Les équipes sont de moins en moins stables du fait de l'accroissement du turnover. »

Quelles que soient ces pratiques, les démissions sont courantes et engendrent un turnover important au sein des cabinets d'affaires, en particulier lorsque les collaborateurs n'espèrent plus être associés au cabinet.

\footnotetext{
7 Ce constat est partagé par la profession et met en question son attractivité pour les jeunes générations (CNB, 2007).
} 


\section{DES PERSPECTIVES} DE CARRIĖRE LIMITÉES

Dans le modèle traditionnel de collaboration libérale, l'accès à l'association se faisait assez naturellement au bout de 7 à 8 ans. Aujourd'hui, cet accès est de plus en plus long (plutôt 10-11 ans), sinon impossible, ce qui décourage certains collaborateurs et les conduit à quitter la profession. Face à ces sorties prématurées de la profession, on peut se demander si la formation professionnelle acquise à l'école des avocats est bien adaptée.

\section{Une indépendance économique difficile à atteindre}

Comme nous l'avons évoqué, la possibilité pour le collaborateur de développer une clientèle est une condition déterminante pour accéder à l'association. Cet apport en clientèle est d'autant plus important dans le cas des associations qui pratiquent la « patrimonialisation de la clientèle ». Cette pratique témoigne du fait que la clientèle est devenue un actif susceptible d'être achetée ou vendue lors de l'entrée ou de la sortie d'une association. Elle constitue un bon indice de l'évolution des cabinets d'avocats vers une logique entrepreneuriale. Notons que celle-ci est de nature différente de celle en œuvre dans les grands cabinets d'affaires où la clientèle est complètement attachée à l'entreprise.

Cette orientation marchande se retrouve dans le mode de rémunération du collaborateur libéral. Le montant de la rétrocession d'honoraires est mis en équivalence avec le droit à la clientèle. Cette rétrocession n'est plus considérée comme un simple défraiement. Elle peut dès lors être d'une certaine manière négociée à la hausse si le collaborateur s'engage à ne pas s'approprier la clientèle du cabinet, et inversement. Mais une rétrocession basse doit être justifiée par le fait qu'on donne les moyens au collaborateur de développer sa clientèle. Sinon, le risque pour le cabinet est une requalification en contrat de travail.

Dans les faits, au-delà des arrangements contractuels entre les parties, des ajustements s'opèrent suivant les contextes et l'existence de normes implicites de régulation des tensions. Il n'en reste pas moins que les quatre collaborateurs interrogés dénoncent le manque de moyens, et surtout de temps, mis à leur disposition pour développer une clientèle personnelle.

Autre aspect : le passage à l'association (payant dans les cabinets où la clientèle est patrimonialisée) où la mise à son compte est d'autant plus problématique que le collaborateur ne dispose pas de capitaux familiaux ${ }^{8}$. On peut mentionner le cas de cette collaboratrice qui envisage, au bout de deux expériences dans des cabinets d'affaires, soit de travailler dans une entreprise, soit de se mettre à son compte :

" Je pourrais, avec des amis, créer ma propre structure, ce qui serait beaucoup plus motivant. Mais je n'ai pas un projet très précis, très mûri, juste un horizon plus excitant. De toute façon, l'installation coûte très cher, il y a le coût du loyer, de l'administratif... Simplement pour mettre en ouvre une société de moyens, il faut au moins assurer 10000 euros d'honoraires/mois. »

Cette mise à son compte est d'autant plus coûteuse aujourd'hui qu'il faut rapidement constituer une structure générant du chiffre d'affaires pour bénéficier d'économies d'échelle sur des investissements de plus en plus importants (en particulier dans les NTIC). Le métier d'avocat peut difficilement à présent s'exercer en solitaire ; l'avocat doit être assisté d'un personnel administratif et de collaborateurs dans un droit de plus en plus complexe.

Pour les collaborateurs en droit des affaires, les difficultés décrites d'accès à l'association sont sans doute moins importantes que dans les domaines du droit où la clientèle est constituée par des particuliers, comme le droit de la famille pour les affaires de divorce. Néanmoins, parmi les cabinets d'affaires, il faut distinguer les très grosses structures d'exercice des petits et moyens cabinets au sein desquels les perspectives de carrière sont plus incertaines, en particulier lorsque ces cabinets ne fournissent pas à leurs collaborateurs les moyens de développer leur propre clientèle, en contrepartie d'une rétrocession relative-

\footnotetext{
${ }^{8}$ Un article des Echos du 4 juillet 2006 confirme les difficultés rencontrées par les petits cabinets pour se pérenniser. Il fait état de 50 procédures collectives en cours au Barreau de Paris dans le cadre de la loi de sauvegarde des entreprises de juillet 2005, qui s'applique au niveau national et pour toutes les activités.
} 
ment importante. D'après nos jeunes interlocuteurs, il y aurait une forme de stigmatisation pour les collaborateurs qui, au bout de dix ans, ne sont pas passés à l'association. Ils sont alors obligés de se « recaser en entreprise ou d'exercer un autre métier s'ils veulent continuer à travailler ».

Cette crise du statut du collaborateur, particulièrement à Paris, est fortement imputée au fonctionnement de l'ordre professionnel, accusé d'entretenir une pléthore de jeunes avocats en ne limitant pas l'entrée à l'école du Barreau. Cette surabondance de jeunes débutants accroîtrait donc la concurrence sur le marché du travail et participerait à la dégradation de leurs conditions d'emploi. Par ailleurs, les études prospectives montrent que les départs à la retraite vont s'accélérer, pour passer de 300 départs à 1000 par an, après 2010 (CNB, Commission prospective, 2007). La profession va donc être confrontée à un véritable problème d'attractivité.

\section{Des avocats qui peinent à se reconvertir}

Nous avons déjà évoqué l'effet de stigmatisation des collaborateurs qui ne deviennent pas associés au bout de dix ans. Un autre inconvénient, pour ceux qui sont habitués à percevoir une rémunération relativement élevée, c'est la difficulté d'accepter une baisse de revenu dans un autre cabinet à Paris et surtout en province. De plus, les avocats de province peuvent avoir un a priori défavorable vis-à-vis de jeunes collaborateurs parisiens.

Plusieurs de nos interlocuteurs ont dénoncé le modèle de collaboration en œuvre dans les cabinets d'affaires, et en particulier la formation insuffisante qu'ils dispensent aux jeunes collaborateurs, le turnover important, ainsi que la dégradation de la qualité de la prestation de service. Certains dénoncent même le fait que les collaborateurs issus de cabinets d'affaires ont pris de mauvaises habitudes, et cela très tôt depuis leur sortie de l'école, et qu'il est alors difficile de les former à un autre état d'esprit. Par ailleurs, ils sont plus spécialistes que généralistes et, pour ceux qui ont travaillé dans des cabinets d'affaires, peu habitués à plaider.

Les difficultés de reconversion ne sont pas uniquement le lot des collaborateurs qui ont commencé leur carrière dans des cabinets d'affaires. Plus généralement, la spécialisation dans un domaine d'activité réduit la mobilité professionnelle des collaborateurs. Mais de façon encore plus générale, les difficultés croissantes d'exercice du métier, d'accès à l'association, conjuguées à des facteurs extra-professionnels (valorisation de la vie familiale et sociale), peuvent expliquer les abandons de l'exercice de la profession. Notons que, statistiquement, un quart des avocats (un tiers pour les femmes) a quitté définitivement la profession au cours des dix premières années après la prestation de serment (Observatoire du CNB, 2007). C'est entre deux et six ans d'activité que les taux de cessation sont les plus importants. Mais l'Observatoire du CNB révèle aussi que les taux de départ sont en augmentation au cours de la période 1994-2005. Cette évolution récente témoigne non seulement des difficultés d'insertion des collaborateurs, mais aussi de mauvaises anticipations de carrière à l'entrée des centres de formation car les cessations définitives interviennent de plus en plus tôt.

Il faudrait disposer des statistiques précises concernant la nature des sorties, mais une grande part des avocats cessionnaires se dirige vers les entreprises. Quel que soit le motif, ces sorties prématurées sont d'autant plus problématiques que la profession supporte une grande part des coûts de formation des avocats ${ }^{9}$. C'est donc aussi le système de formation qui est remis en cause.

\section{Une formation professionnelle inadéquate}

Comme dans beaucoup d'autres secteurs d'activité, l'entrée des jeunes dans la profession d'avocat est de plus en plus tardive (Observatoire du CNB, 2007). Elle correspond à l'allongement de la formation initiale, en particulier par le suivi d'un double cursus ou encore la spécialisation dans un domaine du droit (ancien DESS - diplôme d'études supérieures spécialisées - aujourd'hui M2 professionnel ou doctorat). L'augmentation progressive du niveau de diplôme à l'entrée, qui renforce la sélection des avocats sur leurs aptitudes générales, a accru le risque

\footnotetext{
${ }^{9}$ Rappelons qu'actuellement l'État ne finance la formation initiale des avocats qu'à hauteur de $10 \%$, ce qui est jugé insuffisant par certains syndicats, notamment le Syndicat des Avocats de France.
} 
que le « bon étudiant» ne soit pas forcément un «bon professionnel ». C'est pour réduire ce risque que la réforme du système de 2004 a opté pour une formation professionnelle plus spécialisée et plus appliquée, avec une période de stage pratique d'au moins 6 mois sur un total de 18 mois passés dans le Centre régional de formation de la profession d'avocat. Signalons que le niveau de la « maîtrise » (M1 dans le LMD licence/master/doctorat actuel) est toujours exigé pour présenter l'examen d'entrée et que les doctorants ne sont plus admis sur titre.

Les avocats, qui ont participé à l'élaboration de cette réforme, ne sont pas totalement satisfaits du nouveau système de formation car ils constatent toujours une certaine inadéquation de ce système avec la réalité professionnelle. Malgré la recherche d'une plus grande efficacité de la formation dispensée à l'école des avocats, ils soulignent la trop grande importance accordée aux diplômes par les étudiants ${ }^{10}$, au détriment de l'acquisition de compétences plus professionnelles, en particulier par des stages en entreprise. Un interlocuteur, exerçant dans un petit cabinet parisien en droit social, prône l'instauration d'un système d'apprentissage en alternance basé sur des engagements forts des acteurs. Il ne suffit pas de réaliser des stages « lâchés dans la nature », mais il faut aussi poser la question de l'engagement du formateur et du contrôle de la formation qu'il dispense ${ }^{11}$.

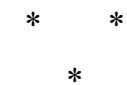

Ainsi, les incertitudes qui entourent les carrières des collaborateurs de même que leur sortie prématurée de la profession sont liées à la remise en cause progressive du fonctionnement d'un marché profes-

\footnotetext{
${ }^{10}$ Notons que cette « course aux diplômes » est encouragée par les cabinets d'affaires qui exigent de plus en plus un double cursus de formation, contrainte qui est d'ailleurs relayée par les pratiques de recrutement des cabinets spécialisés dans les services juridiques (Favereau et alii, 2008).

${ }^{11}$ Il défend d'ailleurs la position du Syndicat des Avocats de France, dont il est membre, qui milite plus généralement pour une formation en alternance afin de donner à l'enseignement dispensé un caractère plus pratique. Cette alternance pourrait se concevoir dans le cadre légal du contrat de professionnalisation, ce qui permettrait également aux structures d'exercice de bénéficier des aides régionales et nationales propres à ce type de contrat.
}

sionnel ${ }^{12}$. Les facteurs d'explication sont multiples : explosion démographique, plus forte hétérogénéité de la profession qui accroît les problèmes de coordination, concurrence internationale dans le domaine du conseil de plus en plus vive, d'une part pour recruter des « jeunes talents », et d'autre part pour attirer de nouveaux clients. Cette évolution a créé non seulement de fortes disparités de rémunération entre les collaborateurs, mais aussi un moindre investissement des cabinets dans leur formation générale, des pratiques de débauchage peu contrôlées par la profession et l'impossibilité pour une part croissante de collaborateurs d'accéder à l'association. Bien sûr, le problème de formation se pose pour les quatre « modèles de collaboration ", mais tous ne génèrent pas les mêmes tensions quant à la possibilité d'existence d'un marché professionnel.

Les modèles du « collaborateur traditionnel » et du « collaborateur innovant » participent à l'entretien du marché professionnel, le second se distinguant du premier par un niveau accru de compétences, ou un éventail plus large, permettant l'innovation dans des domaines du droit pointus. La rétrocession d'honoraires y est plus élevée, de même que le temps d'accès à l'association.

Les cabinets qui sont le plus soumis aux pressions du marché en amont sont les plus à même de déséquilibrer l'ensemble de la profession, en cherchant légitimement à résoudre le problème à leur niveau. Il s'agit en général des cabinets d'avocats d'affaires et, parmi eux, des plus grosses structures. D'un côté, ils offrent un label précieux permettant de débaucher les candidats les plus brillants sans avoir à les former, mais, de l'autre, ils rendent particulièrement difficile la constitution d'une clientèle par un jeune collaborateur. Une solution est alors de l'intégrer dans l'organisation sous un statut salarial.

Les cabinets d'affaires moins réputés ou de plus petite taille n'offrent pas vraiment de modèle de collaboration car les termes du contrat ne sont pas propices à la coopération. La rémunération, tout en restant inférieure à celle pratiquée par les plus grands cabinets, peut atteindre un niveau relativement élevé susceptible d'attirer de nouveaux collaborateurs, mais au

\footnotetext{
12 Cette évolution n'est pas propre au cas français et on la retrouve par exemple au Barreau du Québec (2008).
} 
prix de conditions de travail pénibles. Cette « exploitation » des jeunes avocats a pour conséquence d'accroître leur turnover et, à moyen terme, leur sortie de la profession pour un poste de juriste d'entreprise.

L'intensification des pressions du marché aurait pour risque l'instauration d'un dualisme entre un petit nombre de firmes de très grande taille et une multitude de petits cabinets. Le «marché » ne produit donc pas plus de concurrence et peut être à l'origine d'une grande disparité de revenu entre les avocats.

Face à cette évolution qui engendre des contrats de collaboration de plus en plus marchands, soutenue en cela par l'activité de recruteurs spécialisés, une solution pour les cabinets est de créer leur propre marché interne en salariant leurs collaborateurs. Cela leur permet d'investir massivement dans la formation de leurs collaborateurs dans un domaine du droit pour les fidéliser par des systèmes de rémunération et de promotion adéquats, à l'exemple des avocats salariés qui accèdent à une activité de management. Cette solution salariale apparaît dans la pratique actuelle comme une exception propre à certains cabinets de niche, alors qu'elle est plus développée dans certaines law firms américaines (Goreman, 1999). Elle n'a plus le vent en poupe, et aujourd'hui certains experts de la profession militent pour un statut de collaborateur non salarié, basé sur l'idée de "parasubordination », au sens où le contrat de travail n'est pas fondé sur une relation stricte de subordination de l'employé à l'employeur (Barthélémy et alii, 2008).

Si ces experts s'inscrivent dans l'esprit de la loi Dutreil de 2005 qui vise à limiter la collaboration salariée, ils s'en différencient par l'idée que la création d'un nouveau contrat de collaboration non salariée ne se suffit pas à elle-même. Elle doit être accompagnée d'une négociation collective et prendre en compte l'interdépendance des différentes dimensions de la relation de travail et des règles de concurrence au sein de la profession.

\section{Bibliographie}

Barreau du Québec, (2008), Rapport du Comité sur les problématiques actuelles de la pratique privée.

Barthelemy J., Idrac C., Magnier J.-L., Vielle V. (2008), "Réflexions et propositions à propos d'un statut de l'avocat collaborateur », La semaine juridique-Edition Générale, n ${ }^{\circ}$ 37, pp. 11-17.

Becker G.S. (1964) (1993), Human Capital: A Theoretical and Empirical Analysis, with Special Reference to Education. Chicago, University of Chicago Press, 268 p.

Conseil national des Barreaux (2005), Rapport sur le statut de collaborateur libéral.

Conseil national des Barreaux (2007), Faut-il renforcer l'attractivité de la profession d'avocat? Commission Prospective.
Conseil national des Barreaux (Observatoire) (2007), Regards sur une nouvelle génération d'avocats, septembre.

Conseil national des Barreaux (Observatoire) (2008), Avocats : faits et chiffres. Une profession qui avance, octobre.

Eymard-Duvernay F., Favereau O., Orléan A., Salais R., Thévenot L. (2006), « Valeur, coordination et rationalité : trois thèmes mis en relation par l'économie des conventions ", in Eymard-Duvernay, F. (éd.), L'économie des conventions - méthodes et résultats, Tome I : Débats, Paris, La Découverte, collection « Recherches », pp. 23-44.

Favereau O. (directeur scientifique), Bessis F., Bessy C., Chaserant C., Harnay S., Karpik L., Lazega E. 
(2008), Les avocats entre ordre professionnel et ordre marchand - Concurrence par la qualité et socioéconomie d'une réglementation professionnelle, rapport pour le $\mathrm{CNB}$, septembre.

Goreman O. H. (1999), "Moving away from up or out: Determinants of permanent employment in law firms", Law \& Society Review, vol. 33, n 3, pp. 637-666.

Karpik L. (2007), L'économie des singularités, Paris, Gallimard.

Kerr C. (1954), "The Balkanization of the Labor Markets", in Labor Mobility of Economic Opportunity, E. Bakke.
Lazega E. (2001), The collegial phenomenon: the social mechanisms of cooperation among peers in a corporate law partnership, Oxford, Oxford university press.

Malos S. M., Campion M. A. (1995), "An optionbased model of career mobility in professional service firms", Academy of management Review, vol. 20, $\mathrm{n}^{\circ} 3$, pp. 611-644.

Marsden D. (1999), A theory of Employment systems, Oxford University Press, Oxford.

Vauchez A., Willemez L. (2002), Contribution à la connaissance statistique de la profession d'avocat, Rapport final de l'enquête pour le CNB.

\section{Résumé}

\section{Les avocats, un marché professionnel déstabilisé \\ Christian Bessy}

Les économistes montrent qu'un marché professionnel du travail est une construction fragile faite de relations de coopération et de concurrence entre employeurs. Un des problèmes est celui du financement de la formation des jeunes professionnels, dès lors que leurs compétences sont aisément transférables d'une entreprise à une autre. À partir d'une enquête auprès de différents cabinets d'avocats, nous montrons que cette profession n'arrive plus véritablement à gérer cette tension liée au financement de la formation et que, par ailleurs, elle est confrontée à des sorties de plus en plus prématurées de son périmètre d'activité. Nous analysons les facteurs à l'origine de cette déstabilisation du marché professionnel, et, en particulier, la plus grande variété des cabinets d'avocats et l'émergence de nouveaux modèles de collaboration qui remettent en cause le modèle traditionnel d'apprentissage et des carrières des avocats. Nous examinons en particulier leurs difficultés d'accès à l'indépendance économique et les freins à leur mobilité.

Mots clefs :

Avocat, marché du travail, cheminement professionnel, formation professionnelle.

Journal of Economic Literature: J 44 